\title{
Reaction pathways and free energy barriers for alkaline hydrolysis of insecticide 2-trimethylammonioethyl methylphosphonofluoridate and related organophosphorus compounds: Electrostatic and steric effects
}

\author{
Ying Xiong and Chang-Guo Zhan* \\ Key Laboratory of Pesticide \& Chemical Biology of the Ministry of Education, College \\ of Chemistry, Central China Normal University, Wuhan 430079, P. R. China and \\ Department of Pharmaceutical Sciences, College of Pharmacy, University of \\ Kentucky, 725 Rose Street, Lexington, KY 40536 \\ zhan@uky.edu
}

Supporting Information Available. Cartesian coordinates of the geometries optimized at the HF/6-31+G(d) level, along with their total energies and numbers of imaginary vibrational frequencies. This material is available free of charge via the Internet at http://pubs.acs.org.

Energies in Hartree:

Reactant-1

\begin{tabular}{cccccc} 
Center & Atomic & Atomic & \multicolumn{3}{c}{ Coordinates (Angstroms) } \\
Number & Number & Type & X & $Y$ & $Z$ \\
-1 & 15 & 0 & -1.881767 & -0.159941 & 0.134916 \\
2 & 6 & 0 & -3.005349 & -0.974113 & -0.981502 \\
3 & 8 & 0 & -1.239200 & -0.929084 & 1.188582 \\
4 & 8 & 0 & -0.821396 & 0.566915 & -0.803152 \\
5 & 6 & 0 & 0.150605 & 1.442996 & -0.258053 \\
6 & 6 & 0 & 1.564603 & 1.061398 & -0.663575 \\
7 & 7 & 0 & 2.212222 & -0.140235 & -0.010802 \\
8 & 6 & 0 & 3.663013 & -0.103725 & -0.380967 \\
9 & 6 & 0 & 1.634840 & -1.433352 & -0.507602 \\
10 & 6 & 0 & 2.094883 & -0.095830 & 1.483868 \\
11 & 9 & 0 & -2.697487 & 1.042125 & 0.689589 \\
12 & 1 & 0 & -3.408521 & -0.269245 & -1.697103 \\
13 & 1 & 0 & -3.815165 & -1.405818 & -0.404991 \\
14 & 1 & 0 & -2.489963 & -1.769350 & -1.505740 \\
15 & 1 & 0 & -0.030038 & 2.420635 & -0.683032 \\
16 & 1 & 0 & 0.044388 & 1.524607 & 0.813907 \\
17 & 1 & 0 & 2.199759 & 1.904128 & -0.425573 \\
18 & 1 & 0 & 1.607242 & 0.895767 & -1.730594 \\
& & & & &
\end{tabular}

\footnotetext{
* Corresponding author
} 


$\begin{array}{rrrrrr}19 & 1 & 0 & 4.152095 & -0.971750 & 0.032803 \\ 20 & 1 & 0 & 4.111544 & 0.792328 & 0.020426 \\ 21 & 1 & 0 & 3.755042 & -0.111642 & -1.456836 \\ 22 & 1 & 0 & 2.243760 & -2.240075 & -0.128326 \\ 23 & 1 & 0 & 1.656618 & -1.434529 & -1.586926 \\ 24 & 1 & 0 & 0.631240 & -1.541067 & -0.139711 \\ 25 & 1 & 0 & 2.716388 & -0.877120 & 1.894252 \\ 26 & 1 & 0 & 1.070290 & -0.266930 & 1.771317 \\ 27 & 1 & 0 & 2.442872 & 0.864800 & 1.835256\end{array}$

Total energy: $\quad-880.742994$

Number of imaginary frequencies: 0

\section{OH-}

\begin{tabular}{|c|c|c|c|c|c|}
\hline \multirow{2}{*}{$\begin{array}{l}\text { Center } \\
\text { Number }\end{array}$} & \multirow{2}{*}{$\begin{array}{l}\text { Atomic } \\
\text { Number }\end{array}$} & \multirow{2}{*}{$\begin{array}{l}\text { Atomic } \\
\text { Type }\end{array}$} & \multicolumn{3}{|c|}{ Coordinates (Angstroms) } \\
\hline & & & $x$ & $\mathrm{Y}$ & Z \\
\hline 1 & 8 & 0 & 0.000000 & 0.000000 & 0.105836 \\
\hline 2 & 1 & 0 & 0.000000 & 0.000000 & -0.846692 \\
\hline
\end{tabular}

Total energy: -75.376420

Number of imaginary frequencies: 0

\section{HBR-1A}

\begin{tabular}{|c|c|c|c|c|c|}
\hline \multirow{2}{*}{$\begin{array}{l}\text { Center } \\
\text { Number }\end{array}$} & \multirow{2}{*}{$\begin{array}{l}\text { Atomic } \\
\text { Number }\end{array}$} & \multirow{2}{*}{$\begin{array}{l}\text { Atomic } \\
\text { Type }\end{array}$} & \multicolumn{3}{|c|}{ Coordinates (Angstroms) } \\
\hline & & & $X$ & $\mathrm{Y}$ & Z \\
\hline 1 & 15 & 0 & -2.250741 & -0.193995 & -0.039366 \\
\hline 2 & 9 & 0 & -2.852061 & -1.258462 & 0.940726 \\
\hline 3 & 6 & 0 & -2.359450 & 1.327260 & 0.885732 \\
\hline 4 & 8 & 0 & -0.740026 & -0.681522 & 0.011118 \\
\hline 5 & 6 & 0 & 0.146008 & -0.401789 & -1.072362 \\
\hline 6 & 6 & 0 & 1.478742 & -1.050436 & -0.749443 \\
\hline 7 & 7 & 0 & 2.427917 & -0.267875 & 0.126072 \\
\hline 8 & 6 & 0 & 3.541505 & -1.177973 & 0.493445 \\
\hline 9 & 6 & 0 & 1.759002 & 0.230012 & 1.377602 \\
\hline 10 & 6 & 0 & 2.979441 & 0.914611 & -0.621182 \\
\hline 11 & 8 & 0 & -2.852575 & -0.233295 & -1.359416 \\
\hline 12 & 1 & 0 & -0.241075 & -0.877461 & -1.963315 \\
\hline 13 & 1 & 0 & 0.219861 & 0.669961 & -1.208520 \\
\hline 14 & 1 & 0 & 2.018575 & -1.237435 & -1.668206 \\
\hline 15 & 1 & 0 & 1.303081 & -1.996086 & -0.255599 \\
\hline
\end{tabular}




$\begin{array}{rrrrrr}16 & 1 & 0 & 4.268894 & -0.629973 & 1.073021 \\ 17 & 1 & 0 & 4.007892 & -1.557565 & -0.404709 \\ 18 & 1 & 0 & 3.156548 & -1.999741 & 1.079817 \\ 19 & 1 & 0 & 2.535029 & 0.615362 & 2.024817 \\ 20 & 1 & 0 & 1.257467 & -0.600142 & 1.851964 \\ 21 & 1 & 0 & 1.077866 & 1.026988 & 1.104695 \\ 22 & 1 & 0 & 3.745840 & 1.363195 & -0.004673 \\ 23 & 1 & 0 & 2.170143 & 1.627782 & -0.772682 \\ 24 & 1 & 0 & 3.418009 & 0.558385 & -1.543613 \\ 25 & 8 & 0 & 0.467039 & 2.399226 & -0.248880 \\ 26 & 1 & 0 & 0.345531 & 3.329977 & -0.381252 \\ 27 & 1 & 0 & -1.595236 & 2.001141 & 0.503915 \\ 28 & 1 & 0 & -3.349317 & 1.751268 & 0.763678 \\ 29 & 1 & 0 & -2.171855 & 1.140178 & 1.936769\end{array}$

Total energy: $\quad-956.300280$

Number of imaginary frequencies: 0

\section{TS1-1A}

\begin{tabular}{|c|c|c|c|c|c|}
\hline \multirow{2}{*}{$\begin{array}{l}\text { Center } \\
\text { Number }\end{array}$} & \multirow{2}{*}{$\begin{array}{l}\text { Atomic } \\
\text { Number }\end{array}$} & \multirow{2}{*}{$\begin{array}{l}\text { Atomic } \\
\text { Type }\end{array}$} & \multicolumn{3}{|c|}{ Coordinates (Angstroms) } \\
\hline & & & $x$ & $\mathrm{Y}$ & Z \\
\hline 1 & 15 & 0 & 2.011417 & -0.065733 & 0.041745 \\
\hline 2 & 9 & 0 & 2.879253 & -1.121572 & -0.801252 \\
\hline 3 & 6 & 0 & 2.354700 & 1.318936 & -1.056942 \\
\hline 4 & 8 & 0 & 0.621564 & -0.790902 & -0.307628 \\
\hline 5 & 6 & 0 & -0.257468 & -1.164024 & 0.718982 \\
\hline 6 & 6 & 0 & -1.664359 & -1.280348 & 0.161783 \\
\hline 7 & 7 & 0 & -2.415637 & -0.004930 & -0.138777 \\
\hline 8 & 6 & 0 & -3.766242 & -0.396439 & -0.616724 \\
\hline 9 & 6 & 0 & -1.737565 & 0.809171 & -1.205850 \\
\hline 10 & 6 & 0 & -2.548609 & 0.841440 & 1.093598 \\
\hline 11 & 8 & 0 & 2.485471 & -0.130966 & 1.416168 \\
\hline 12 & 1 & 0 & 0.006469 & -2.159037 & 1.062731 \\
\hline 13 & 1 & 0 & -0.188180 & -0.471006 & 1.538550 \\
\hline 14 & 1 & 0 & -2.273775 & -1.814725 & 0.879792 \\
\hline 15 & 1 & 0 & -1.643285 & -1.846740 & -0.759407 \\
\hline 16 & 1 & 0 & -4.335513 & 0.494196 & -0.835930 \\
\hline 17 & 1 & 0 & -4.270031 & -0.966424 & 0.150621 \\
\hline 18 & 1 & 0 & -3.671543 & -0.993972 & -1.512013 \\
\hline 19 & 1 & 0 & -2.423551 & 1.592285 & -1.498932 \\
\hline 20 & 1 & 0 & -1.530541 & 0.165068 & -2.048037 \\
\hline 21 & 1 & 0 & -0.837948 & 1.234080 & -0.786763 \\
\hline
\end{tabular}




$\begin{array}{lllrrr}22 & 1 & 0 & -3.227726 & 1.651300 & 0.866515 \\ 23 & 1 & 0 & -1.563487 & 1.232092 & 1.331358 \\ 24 & 1 & 0 & -2.962987 & 0.231347 & 1.885029 \\ 25 & 8 & 0 & 0.320227 & 1.571911 & 0.781003 \\ 26 & 1 & 0 & 0.772625 & 2.048814 & 1.463837 \\ 27 & 1 & 0 & 2.182775 & 2.253259 & -0.554184 \\ 28 & 1 & 0 & 3.383333 & 1.230912 & -1.387421 \\ 29 & 1 & 0 & 1.707459 & 1.260449 & -1.924650\end{array}$

Total energy: -956.294473

Number of imaginary frequencies: 1

\section{INT-1A}

\begin{tabular}{|c|c|c|c|c|c|}
\hline \multirow{2}{*}{$\begin{array}{l}\text { Center } \\
\text { Number }\end{array}$} & \multirow{2}{*}{$\begin{array}{l}\text { Atomic } \\
\text { Number }\end{array}$} & \multirow{2}{*}{$\begin{array}{l}\text { Atomic } \\
\text { Type }\end{array}$} & \multicolumn{3}{|c|}{ Coordinates (Angstroms) } \\
\hline & & & $x$ & $\mathrm{Y}$ & Z \\
\hline 1 & 15 & 0 & 1.629524 & -0.125846 & 0.149668 \\
\hline 2 & 9 & 0 & 1.560241 & -1.591802 & -0.727553 \\
\hline 3 & 6 & 0 & 3.418706 & -0.127897 & -0.198039 \\
\hline 4 & 8 & 0 & 0.633054 & 0.408136 & -1.065620 \\
\hline 5 & 6 & 0 & -0.340811 & 1.379275 & -0.896497 \\
\hline 6 & 6 & 0 & -1.304205 & 1.075105 & 0.247395 \\
\hline 7 & 7 & 0 & -2.237989 & -0.115456 & 0.067011 \\
\hline 8 & 6 & 0 & -2.863533 & -0.377084 & 1.393414 \\
\hline 9 & 6 & 0 & -1.531241 & -1.376602 & -0.362482 \\
\hline 10 & 6 & 0 & -3.298077 & 0.204225 & -0.921333 \\
\hline 11 & 8 & 0 & 0.988960 & -0.674275 & 1.388850 \\
\hline 12 & 8 & 0 & 1.726009 & 1.523972 & 0.699713 \\
\hline 13 & 1 & 0 & 3.951728 & 0.435363 & 0.555687 \\
\hline 14 & 1 & 0 & 3.797474 & -1.137608 & -0.258229 \\
\hline 15 & 1 & 0 & 3.578922 & 0.360376 & -1.154751 \\
\hline 16 & 1 & 0 & 0.089751 & 2.343706 & -0.669583 \\
\hline 17 & 1 & 0 & -0.862315 & 1.457467 & -1.845163 \\
\hline 18 & 1 & 0 & -1.955478 & 1.925259 & 0.410992 \\
\hline 19 & 1 & 0 & -0.735365 & 0.878520 & 1.138701 \\
\hline 20 & 1 & 0 & -3.582231 & -1.177918 & 1.295149 \\
\hline 21 & 1 & 0 & -3.359931 & 0.518973 & 1.737855 \\
\hline 22 & 1 & 0 & -2.086453 & -0.660895 & 2.086495 \\
\hline 23 & 1 & 0 & -2.275726 & -2.160901 & -0.391945 \\
\hline 24 & 1 & 0 & -0.749552 & -1.598344 & 0.345106 \\
\hline 25 & 1 & 0 & -1.092375 & -1.229542 & -1.332946 \\
\hline 26 & 1 & 0 & -3.976306 & -0.633339 & -0.995933 \\
\hline 27 & 1 & 0 & -2.848501 & 0.384825 & -1.884516 \\
\hline
\end{tabular}




$\begin{array}{lllrrc}28 & 1 & 0 & -3.839218 & 1.082294 & -0.598807 \\ 29 & 1 & 0 & 1.857242 & 1.509073 & 1.637479\end{array}$

Total energy: -956.314251

Number of imaginary frequencies: 0

\section{TS2-1A}

\begin{tabular}{|c|c|c|c|c|c|}
\hline \multirow{2}{*}{$\begin{array}{l}\text { Center } \\
\text { Number }\end{array}$} & \multirow{2}{*}{$\begin{array}{l}\text { Atomic } \\
\text { Number }\end{array}$} & \multirow{2}{*}{$\begin{array}{l}\text { Atomic } \\
\text { Type }\end{array}$} & \multicolumn{3}{|c|}{ Coordinates (Angstroms) } \\
\hline & & & $X$ & Y & Z \\
\hline 1 & 15 & 0 & 1.717642 & -0.055628 & 0.155052 \\
\hline 2 & 9 & 0 & 0.624088 & -1.573652 & -0.012885 \\
\hline 3 & 6 & 0 & 2.927699 & -1.125780 & -0.670651 \\
\hline 4 & 8 & 0 & 0.631876 & 0.560651 & -0.924093 \\
\hline 5 & 6 & 0 & -0.256110 & 1.532518 & -0.491239 \\
\hline 6 & 6 & 0 & -1.266685 & 0.995103 & 0.518911 \\
\hline 7 & 7 & 0 & -2.258158 & -0.069088 & 0.025385 \\
\hline 8 & 6 & 0 & -2.279628 & -1.174935 & 1.036228 \\
\hline 9 & 6 & 0 & -1.914252 & -0.675411 & -1.305827 \\
\hline 10 & 6 & 0 & -3.610443 & 0.535337 & -0.075326 \\
\hline 11 & 8 & 0 & 1.411995 & 0.025264 & 1.609353 \\
\hline 12 & 8 & 0 & 2.670277 & 1.337440 & 0.020303 \\
\hline 13 & 1 & 0 & 3.908656 & -0.673742 & -0.646380 \\
\hline 14 & 1 & 0 & 2.935272 & -2.094520 & -0.192936 \\
\hline 15 & 1 & 0 & 2.624614 & -1.263405 & -1.702695 \\
\hline 16 & 1 & 0 & 0.260891 & 2.343816 & 0.010960 \\
\hline 17 & 1 & 0 & -0.735465 & 1.948922 & -1.370315 \\
\hline 18 & 1 & 0 & -1.866807 & 1.812195 & 0.896137 \\
\hline 19 & 1 & 0 & -0.714483 & 0.556695 & 1.332068 \\
\hline 20 & 1 & 0 & -3.021271 & -1.902685 & 0.737419 \\
\hline 21 & 1 & 0 & -2.541015 & -0.758208 & 1.998193 \\
\hline 22 & 1 & 0 & -1.294744 & -1.615572 & 1.066587 \\
\hline 23 & 1 & 0 & -2.686295 & -1.396809 & -1.535769 \\
\hline 24 & 1 & 0 & -0.949410 & -1.144089 & -1.232348 \\
\hline 25 & 1 & 0 & -1.909534 & 0.098713 & -2.056592 \\
\hline 26 & 1 & 0 & -4.313296 & -0.211706 & -0.414206 \\
\hline 27 & 1 & 0 & -3.582211 & 1.352355 & -0.782223 \\
\hline 28 & 1 & 0 & -3.913450 & 0.903617 & 0.893754 \\
\hline 29 & 1 & 0 & 2.907568 & 1.617507 & 0.893751 \\
\hline
\end{tabular}

Total energy: -956.309484

Number of imaginary frequencies: 1 
Product-1A

\begin{tabular}{|c|c|c|c|c|c|}
\hline \multirow{2}{*}{$\begin{array}{l}\text { Center } \\
\text { Number }\end{array}$} & \multirow{2}{*}{$\begin{array}{l}\text { Atomic } \\
\text { Number }\end{array}$} & \multirow{2}{*}{$\begin{array}{l}\text { Atomic } \\
\text { Type }\end{array}$} & \multicolumn{3}{|c|}{ Coordinates (Angstroms) } \\
\hline & & & $\mathrm{X}$ & $\mathrm{Y}$ & $\mathrm{Z}$ \\
\hline 1 & 15 & 0 & 2.160355 & -0.041868 & -0.015246 \\
\hline 2 & 6 & 0 & 2.781136 & 1.533197 & -0.589016 \\
\hline 3 & 8 & 0 & 0.594827 & 0.047418 & -0.390388 \\
\hline 4 & 6 & 0 & -0.173408 & -1.135121 & -0.409610 \\
\hline 5 & 6 & 0 & -1.593635 & -0.829315 & -0.845835 \\
\hline 6 & 7 & 0 & -2.398517 & 0.111064 & 0.029124 \\
\hline 7 & 6 & 0 & -3.841578 & -0.063588 & -0.329813 \\
\hline 8 & 6 & 0 & -2.024873 & 1.542825 & -0.221889 \\
\hline 9 & 6 & 0 & -2.222653 & -0.195268 & 1.482229 \\
\hline 10 & 8 & 0 & 2.800328 & -1.254174 & -0.498182 \\
\hline 11 & 8 & 0 & 2.098680 & 0.055572 & 1.577062 \\
\hline 12 & 1 & 0 & 3.824242 & 1.619813 & -0.307265 \\
\hline 13 & 1 & 0 & 2.709508 & 1.582854 & -1.668498 \\
\hline 14 & 1 & 0 & 2.230337 & 2.354461 & -0.147473 \\
\hline 15 & 1 & 0 & 0.235104 & -1.830404 & -1.130780 \\
\hline 16 & 1 & 0 & -0.148024 & -1.614916 & 0.559630 \\
\hline 17 & 1 & 0 & -2.136730 & -1.764469 & -0.871915 \\
\hline 18 & 1 & 0 & -1.599920 & -0.402735 & -1.838536 \\
\hline 19 & 1 & 0 & -4.430661 & 0.653323 & 0.221346 \\
\hline 20 & 1 & 0 & -4.153255 & -1.065249 & -0.075568 \\
\hline 21 & 1 & 0 & -3.963797 & 0.101305 & -1.389840 \\
\hline 22 & 1 & 0 & -2.649834 & 2.169900 & 0.395999 \\
\hline 23 & 1 & 0 & -2.196771 & 1.769043 & -1.263403 \\
\hline 24 & 1 & 0 & -0.987892 & 1.686483 & 0.019831 \\
\hline 25 & 1 & 0 & -2.914389 & 0.411870 & 2.046208 \\
\hline 26 & 1 & 0 & -1.212652 & 0.036198 & 1.779455 \\
\hline 27 & 1 & 0 & -2.435245 & -1.240252 & 1.653520 \\
\hline 28 & 1 & 0 & 2.713666 & -0.523557 & 2.017782 \\
\hline
\end{tabular}

Total energy: $\quad-856.735049$

Number of imaginary frequencies: 0

TS1-1B

\begin{tabular}{cccccc}
$\begin{array}{l}\text { Center } \\
\text { Number }\end{array}$ & Atomic & Atomic & \multicolumn{4}{c}{ Coordinates } & \multicolumn{2}{c}{ (Angstroms) } \\
& Number & Type & X & $Y$ & $Z$ \\
\hdashline 1 & 15 & 0 & -1.874618 & 0.037905 & 0.220092 \\
2 & 6 & 0 & -3.113164 & -1.269381 & 0.449914
\end{tabular}




\begin{tabular}{|c|c|c|c|c|c|}
\hline 3 & 8 & 0 & -1.735981 & 0.727962 & 1.500083 \\
\hline 4 & 8 & 0 & -0.722148 & -0.928765 & -0.338164 \\
\hline 5 & 6 & 0 & 0.284768 & -0.762583 & -1.298632 \\
\hline 6 & 6 & 0 & 1.648472 & -1.101489 & -0.712878 \\
\hline 7 & 7 & 0 & 2.344218 & -0.083527 & 0.157647 \\
\hline 8 & 6 & 0 & 3.643070 & -0.675490 & 0.567001 \\
\hline 9 & 6 & 0 & 1.547933 & 0.241348 & 1.393942 \\
\hline 10 & 6 & 0 & 2.597819 & 1.183682 & -0.604665 \\
\hline 11 & 9 & 0 & -2.591008 & 0.724687 & -0.991163 \\
\hline 12 & 1 & 0 & -3.300613 & -1.812911 & -0.469431 \\
\hline 13 & 1 & 0 & -4.040114 & -0.822083 & 0.791495 \\
\hline 14 & 1 & 0 & -2.774153 & -1.965578 & 1.208560 \\
\hline 15 & 1 & 0 & 0.113988 & -1.517059 & -2.060478 \\
\hline 16 & 1 & 0 & 0.236714 & 0.218770 & -1.733455 \\
\hline 17 & 1 & 0 & 2.329406 & -1.293144 & -1.533159 \\
\hline 18 & 1 & 0 & 1.566143 & -2.002645 & -0.120103 \\
\hline 19 & 1 & 0 & 4.177382 & 0.035887 & 1.178440 \\
\hline 20 & 1 & 0 & 4.228849 & -0.907727 & -0.310981 \\
\hline 21 & 1 & 0 & 3.464744 & -1.576651 & 1.135789 \\
\hline 22 & 1 & 0 & 2.184260 & 0.828220 & 2.042049 \\
\hline 23 & 1 & 0 & 1.278832 & -0.684153 & 1.881254 \\
\hline 24 & 1 & 0 & 0.675022 & 0.805524 & 1.102434 \\
\hline 25 & 1 & 0 & 3.203590 & 1.827633 & 0.017640 \\
\hline 26 & 1 & 0 & 1.636415 & 1.646415 & -0.807007 \\
\hline 27 & 1 & 0 & 3.142174 & 0.935849 & -1.506460 \\
\hline 28 & 8 & 0 & -0.296220 & 1.655460 & -0.520356 \\
\hline 29 & 1 & 0 & -0.712414 & 2.443811 & -0.201634 \\
\hline
\end{tabular}

Total energy: -956.279607

Number of imaginary frequencies: 1

\section{Reactant-2}

\begin{tabular}{|c|c|c|c|c|c|}
\hline \multirow{2}{*}{$\begin{array}{l}\text { Center } \\
\text { Number }\end{array}$} & \multirow{2}{*}{$\begin{array}{l}\text { Atomic } \\
\text { Number }\end{array}$} & \multirow{2}{*}{$\begin{array}{l}\text { Atomic } \\
\text { Type }\end{array}$} & \multicolumn{3}{|c|}{ Coordinates (Angstroms) } \\
\hline & & & $\mathrm{X}$ & $\mathrm{Y}$ & $\mathrm{Z}$ \\
\hline 1 & 15 & 0 & -0.353901 & -0.135876 & -0.101000 \\
\hline 2 & 9 & 0 & -0.583713 & -0.747571 & 1.324557 \\
\hline 3 & 6 & 0 & -1.689848 & 1.045114 & -0.228516 \\
\hline 4 & 8 & 0 & 0.921444 & 0.755465 & 0.119998 \\
\hline 5 & 6 & 0 & 2.247736 & 0.247416 & -0.017273 \\
\hline 6 & 8 & 0 & -0.248247 & -1.161381 & -1.120889 \\
\hline 7 & 1 & 0 & 2.480326 & -0.399917 & 0.817057 \\
\hline 8 & 1 & 0 & 2.344596 & -0.292680 & -0.947000 \\
\hline
\end{tabular}




$\begin{array}{rrrrrr}9 & 1 & 0 & -1.595258 & 1.593348 & -1.157702 \\ 10 & 1 & 0 & -2.633743 & 0.513256 & -0.229656 \\ 11 & 1 & 0 & -1.667121 & 1.738869 & 0.602221 \\ 12 & 1 & 0 & 2.900210 & 1.105558 & -0.009061\end{array}$

Total energy: $\quad-669.216545$

Number of imaginary frequencies: 0

\section{HBR-2A}

\begin{tabular}{cccccc} 
Center & Atomic & Atomic & \multicolumn{3}{c}{ Coordinates (Angstroms) } \\
Number & Number & Type & $X$ & $Y$ & $Z$ \\
\hdashline 1 & 15 & 0 & -0.767197 & -0.120661 & 0.136003 \\
2 & 9 & 0 & -2.074574 & -0.209519 & -0.749562 \\
3 & 6 & 0 & 0.127762 & -1.597457 & -0.308933 \\
4 & 8 & 0 & -0.110291 & 1.107696 & -0.587098 \\
5 & 6 & 0 & 1.090559 & 1.731625 & -0.088729 \\
6 & 8 & 0 & -1.108521 & 0.055740 & 1.542672 \\
7 & 1 & 0 & 0.873233 & 2.181782 & 0.871706 \\
8 & 1 & 0 & 1.895876 & 1.006242 & -0.016003 \\
9 & 8 & 0 & 2.971211 & -0.679903 & 0.035655 \\
10 & 1 & 0 & 3.818192 & -0.994730 & 0.324263 \\
11 & 1 & 0 & 1.194487 & -1.459781 & -0.079457 \\
12 & 1 & 0 & -0.279924 & -2.437654 & 0.243724 \\
13 & 1 & 0 & 0.023886 & -1.776903 & -1.373655 \\
14 & 1 & 0 & 1.324255 & 2.503355 & -0.808414 \\
& 1 & & & &
\end{tabular}

Total energy: $\quad-744.633729105$

Number of imaginary frequencies: 0

\section{TS1-2A}

\begin{tabular}{|c|c|c|c|c|c|}
\hline \multirow{2}{*}{$\begin{array}{l}\text { Center } \\
\text { Number }\end{array}$} & \multirow{2}{*}{$\begin{array}{l}\text { Atomic } \\
\text { Number }\end{array}$} & \multirow{2}{*}{$\begin{array}{l}\text { Atomic } \\
\text { Type }\end{array}$} & \multicolumn{3}{|c|}{ Coordinates (Angstroms) } \\
\hline & & & $\mathrm{X}$ & $\mathrm{Y}$ & Z \\
\hline 1 & 15 & 0 & 0.506306 & -0.022264 & 0.159699 \\
\hline 2 & 9 & 0 & 1.843130 & -0.865799 & -0.166798 \\
\hline 3 & 6 & 0 & 0.950788 & 1.412969 & -0.824702 \\
\hline 4 & 8 & 0 & -0.457081 & -0.980388 & -0.636237 \\
\hline 5 & 6 & 0 & -1.782726 & -1.309008 & -0.235213 \\
\hline 6 & 8 & 0 & 0.402863 & 0.045468 & 1.612912 \\
\hline 7 & 1 & 0 & -1.754766 & -1.819848 & 0.720248 \\
\hline 8 & 1 & 0 & -2.355670 & -0.396696 & -0.171982 \\
\hline
\end{tabular}




$\begin{array}{rrrrrr}9 & 8 & 0 & -1.717380 & 1.540567 & 0.026442 \\ 10 & 1 & 0 & -1.889833 & 1.822350 & 0.916331 \\ 11 & 1 & 0 & 0.108023 & 2.087335 & -0.821443 \\ 12 & 1 & 0 & 1.832255 & 1.864077 & -0.378446 \\ 13 & 1 & 0 & 1.196305 & 1.086016 & -1.829874 \\ 14 & 1 & 0 & -2.154675 & -1.986025 & -0.994583\end{array}$

Total energy: $\quad-744.627906$

Number of imaginary frequencies: 1

\section{Reactant-3}

\begin{tabular}{|c|c|c|c|c|c|}
\hline \multirow{2}{*}{$\begin{array}{l}\text { Center } \\
\text { Number }\end{array}$} & \multirow{2}{*}{$\begin{array}{l}\text { Atomic } \\
\text { Number }\end{array}$} & \multirow{2}{*}{$\begin{array}{c}\text { Atomic } \\
\text { Type }\end{array}$} & \multicolumn{3}{|c|}{ Coordinates (Angstroms) } \\
\hline & & & $X$ & $\mathrm{Y}$ & $\mathrm{Z}$ \\
\hline 1 & 15 & 0 & 2.392395 & -0.096016 & 0.108802 \\
\hline 2 & 6 & 0 & 3.445066 & 1.346389 & 0.206298 \\
\hline 3 & 8 & 0 & 2.497814 & -1.085923 & 1.164043 \\
\hline 4 & 8 & 0 & 0.962371 & 0.493127 & -0.156685 \\
\hline 5 & 6 & 0 & -0.238288 & -0.290331 & -0.042244 \\
\hline 6 & 6 & 0 & -1.406411 & 0.679850 & -0.091599 \\
\hline 7 & 6 & 0 & -2.815603 & 0.053303 & 0.005055 \\
\hline 8 & 6 & 0 & -3.834781 & 1.201492 & -0.062188 \\
\hline 9 & 6 & 0 & -3.000045 & -0.694226 & 1.335525 \\
\hline 10 & 6 & 0 & -3.076985 & -0.908161 & -1.165620 \\
\hline 11 & 9 & 0 & 2.775079 & -0.692030 & -1.291368 \\
\hline 12 & 1 & 0 & 3.292162 & 1.990056 & -0.650620 \\
\hline 13 & 1 & 0 & 4.480268 & 1.028002 & 0.238986 \\
\hline 14 & 1 & 0 & 3.218604 & 1.894605 & 1.112483 \\
\hline 15 & 1 & 0 & -0.255420 & -0.990325 & -0.866101 \\
\hline 16 & 1 & 0 & -0.194445 & -0.839480 & 0.885832 \\
\hline 17 & 1 & 0 & -1.331886 & 1.245005 & -1.016709 \\
\hline 18 & 1 & 0 & -1.278927 & 1.392355 & 0.718958 \\
\hline 19 & 1 & 0 & -4.850605 & 0.822283 & 0.001572 \\
\hline 20 & 1 & 0 & -3.745523 & 1.753676 & -0.993696 \\
\hline 21 & 1 & 0 & -3.691824 & 1.903357 & 0.754789 \\
\hline 22 & 1 & 0 & -4.023442 & -1.043918 & 1.436287 \\
\hline 23 & 1 & 0 & -2.787875 & -0.047870 & 2.182998 \\
\hline 24 & 1 & 0 & -2.355102 & -1.564005 & 1.411037 \\
\hline 25 & 1 & 0 & -4.103560 & -1.262291 & -1.144794 \\
\hline 26 & 1 & 0 & -2.435868 & -1.783399 & -1.128996 \\
\hline 27 & 1 & 0 & -2.917391 & -0.417075 & -2.121973 \\
\hline
\end{tabular}

Total energy: $\quad-864.394365$ 
Number of imaginary frequencies: 0

HBR-3A

\begin{tabular}{|c|c|c|c|c|c|}
\hline \multirow{2}{*}{$\begin{array}{l}\text { Center } \\
\text { Number }\end{array}$} & \multirow{2}{*}{$\begin{array}{l}\text { Atomic } \\
\text { Number }\end{array}$} & \multirow{2}{*}{$\begin{array}{l}\text { Atomic } \\
\text { Type }\end{array}$} & \multicolumn{3}{|c|}{ Coordinates (Angstroms) } \\
\hline & & & $\mathrm{X}$ & $\mathrm{Y}$ & Z \\
\hline 1 & 15 & 0 & -2.204068 & -0.284238 & -0.046151 \\
\hline 2 & 9 & 0 & -2.705883 & -1.450909 & 0.895088 \\
\hline 3 & 6 & 0 & -2.305527 & 1.154423 & 1.002543 \\
\hline 4 & 8 & 0 & -0.702023 & -0.723449 & -0.151114 \\
\hline 5 & 6 & 0 & 0.205121 & -0.151859 & -1.126890 \\
\hline 6 & 6 & 0 & 1.560594 & -0.826663 & -0.963680 \\
\hline 7 & 6 & 0 & 2.523054 & -0.272544 & 0.112072 \\
\hline 8 & 6 & 0 & 3.735260 & -1.216625 & 0.176625 \\
\hline 9 & 6 & 0 & 1.858604 & -0.211712 & 1.495492 \\
\hline 10 & 6 & 0 & 2.995614 & 1.138504 & -0.273694 \\
\hline 11 & 8 & 0 & -2.964577 & -0.258549 & -1.289999 \\
\hline 12 & 1 & 0 & -0.203103 & -0.382894 & -2.102688 \\
\hline 13 & 1 & 0 & 0.236224 & 0.921830 & -0.983721 \\
\hline 14 & 1 & 0 & 2.068856 & -0.755177 & -1.925287 \\
\hline 15 & 1 & 0 & 1.386280 & -1.886740 & -0.788048 \\
\hline 16 & 1 & 0 & 4.472033 & -0.854251 & 0.890193 \\
\hline 17 & 1 & 0 & 4.226486 & -1.296404 & -0.791564 \\
\hline 18 & 1 & 0 & 3.442547 & -2.219128 & 0.483062 \\
\hline 19 & 1 & 0 & 2.592393 & 0.076647 & 2.246995 \\
\hline 20 & 1 & 0 & 1.446128 & -1.176186 & 1.782009 \\
\hline 21 & 1 & 0 & 1.063889 & 0.522765 & 1.510631 \\
\hline 22 & 1 & 0 & 3.725135 & 1.499860 & 0.450478 \\
\hline 23 & 1 & 0 & 2.167664 & 1.840548 & -0.296087 \\
\hline 24 & 1 & 0 & 3.482868 & 1.129306 & -1.249064 \\
\hline 25 & 8 & 0 & 0.012798 & 2.757578 & -0.115580 \\
\hline 26 & 1 & 0 & 0.049256 & 3.685562 & -0.309737 \\
\hline 27 & 1 & 0 & -1.610855 & 1.915866 & 0.620370 \\
\hline 28 & 1 & 0 & -3.325858 & 1.523770 & 1.004696 \\
\hline 29 & 1 & 0 & -2.011867 & 0.890585 & 2.012984 \\
\hline
\end{tabular}

Total energy: $\quad-939.814473848$

Number of imaginary frequencies: 0

\section{TS1-3A}

\begin{tabular}{lccccc} 
Center & Atomic & Atomic & & \multicolumn{3}{c}{ Coordinates (Angstroms) } & \\
Number & Number & Type & X & Y & Z
\end{tabular}




\begin{tabular}{|c|c|c|c|c|c|}
\hline 1 & 15 & 0 & -2.055183 & -0.128213 & -0.030990 \\
\hline 2 & 9 & 0 & -2.802490 & -1.431795 & 0.559967 \\
\hline 3 & 6 & 0 & -2.302343 & 0.888120 & 1.430159 \\
\hline 4 & 8 & 0 & -0.618234 & -0.771110 & -0.034472 \\
\hline 5 & 6 & 0 & 0.298514 & -0.663001 & -1.125265 \\
\hline 6 & 6 & 0 & 1.683175 & -1.089631 & -0.656396 \\
\hline 7 & 6 & 0 & 2.534221 & -0.077048 & 0.144447 \\
\hline 8 & 6 & 0 & 3.863229 & -0.770688 & 0.489505 \\
\hline 9 & 6 & 0 & 1.837492 & 0.356353 & 1.442892 \\
\hline 10 & 6 & 0 & 2.818232 & 1.174823 & -0.700828 \\
\hline 11 & 8 & 0 & -2.708293 & 0.181011 & -1.297957 \\
\hline 12 & 1 & 0 & -0.040278 & -1.344470 & -1.898650 \\
\hline 13 & 1 & 0 & 0.267429 & 0.353596 & -1.478291 \\
\hline 14 & 1 & 0 & 2.246660 & -1.354522 & -1.552508 \\
\hline 15 & 1 & 0 & 1.578578 & -2.007476 & -0.079700 \\
\hline 16 & 1 & 0 & 4.518630 & -0.101161 & 1.042296 \\
\hline 17 & 1 & 0 & 4.393607 & -1.083962 & -0.408335 \\
\hline 18 & 1 & 0 & 3.700836 & -1.654758 & 1.103306 \\
\hline 19 & 1 & 0 & 2.514820 & 0.966829 & 2.040028 \\
\hline 20 & 1 & 0 & 1.550439 & -0.505251 & 2.042624 \\
\hline 21 & 1 & 0 & 0.956248 & 0.943676 & 1.224279 \\
\hline 22 & 1 & 0 & 3.512723 & 1.831433 & -0.178287 \\
\hline 23 & 1 & 0 & 1.905040 & 1.730651 & -0.880988 \\
\hline 24 & 1 & 0 & 3.275744 & 0.909083 & -1.654362 \\
\hline 25 & 8 & 0 & -0.507785 & 2.025668 & -0.429068 \\
\hline 26 & 1 & 0 & -0.979668 & 2.515413 & -1.090956 \\
\hline 27 & 1 & 0 & -1.815112 & 1.836149 & 1.270563 \\
\hline 28 & 1 & 0 & -3.372166 & 1.000956 & 1.579163 \\
\hline 29 & 1 & 0 & -1.883996 & 0.375053 & 2.289852 \\
\hline
\end{tabular}

Total energy: $\quad-939.809403$

Number of imaginary frequencies: 1

\section{Reactant-4}

\begin{tabular}{cccccc}
$\begin{array}{c}\text { Center } \\
\text { Number }\end{array}$ & $\begin{array}{c}\text { Atomic } \\
\text { Number }\end{array}$ & $\begin{array}{c}\text { Atomic } \\
\text { Type }\end{array}$ & \multicolumn{4}{c}{ Coordinates (Angstroms) } \\
\hline 1 & 15 & 0 & 2.123467 & 0.135828 & -0.054071 \\
2 & 6 & 0 & 3.130284 & -1.078086 & -0.897122 \\
3 & 8 & 0 & 2.207936 & 1.514317 & -0.498044 \\
4 & 8 & 0 & 0.691104 & -0.505114 & -0.033577 \\
5 & 6 & 0 & -0.493774 & 0.235085 & 0.302456
\end{tabular}




$\begin{array}{rrrrrr}6 & 6 & 0 & -1.690145 & -0.602462 & -0.110456 \\ 7 & 6 & 0 & -3.042907 & -0.006630 & 0.309039 \\ 8 & 6 & 0 & -4.167584 & -0.999469 & 0.000459 \\ 9 & 6 & 0 & -3.325371 & 1.345426 & -0.356139 \\ 10 & 9 & 0 & 2.585557 & -0.027535 & 1.436430 \\ 11 & 1 & 0 & 2.997735 & -2.057776 & -0.455948 \\ 12 & 1 & 0 & 4.171450 & -0.786944 & -0.824547 \\ 13 & 1 & 0 & 2.848624 & -1.113531 & -1.942302 \\ 14 & 1 & 0 & -0.487900 & 0.409849 & 1.371646 \\ 15 & 1 & 0 & -0.455065 & 1.184689 & -0.208717 \\ 16 & 1 & 0 & -1.577717 & -1.586530 & 0.335217 \\ 17 & 1 & 0 & -1.665860 & -0.740753 & -1.188840 \\ 18 & 1 & 0 & -5.128384 & -0.613937 & 0.327939 \\ 19 & 1 & 0 & -4.004415 & -1.950669 & 0.498509 \\ 20 & 1 & 0 & -4.235363 & -1.191210 & -1.067648 \\ 21 & 1 & 0 & -4.305574 & 1.714933 & -0.070563 \\ 22 & 1 & 0 & -3.309716 & 1.255658 & -1.439777 \\ 23 & 1 & 0 & -2.601708 & 2.103948 & -0.076110 \\ 24 & 1 & 0 & -3.023465 & 0.145852 & 1.387890\end{array}$

Total energy : -825.361310576

Number of imaginary frequencies: 0

\section{HBR-4A}

\begin{tabular}{|c|c|c|c|c|c|}
\hline \multirow{2}{*}{$\begin{array}{l}\text { Center } \\
\text { Number }\end{array}$} & \multirow{2}{*}{$\begin{array}{l}\text { Atomic } \\
\text { Number }\end{array}$} & \multirow{2}{*}{$\begin{array}{l}\text { Atomic } \\
\text { Type }\end{array}$} & \multicolumn{3}{|c|}{ Coordinates (Angstroms) } \\
\hline & & & $x$ & $\mathrm{Y}$ & Z \\
\hline 1 & 15 & 0 & 1.972958 & -0.333369 & -0.024819 \\
\hline 2 & 9 & 0 & 2.383718 & -1.506222 & -1.002298 \\
\hline 3 & 6 & 0 & 2.077516 & 1.113770 & -1.061259 \\
\hline 4 & 8 & 0 & 0.462166 & -0.720553 & 0.147264 \\
\hline 5 & 6 & 0 & -0.394055 & -0.087671 & 1.130624 \\
\hline 6 & 6 & 0 & -1.777310 & -0.695750 & 0.981711 \\
\hline 7 & 6 & 0 & -2.500130 & -0.372564 & -0.334809 \\
\hline 8 & 6 & 0 & -2.908980 & 1.101901 & -0.420611 \\
\hline 9 & 8 & 0 & 2.793387 & -0.353259 & 1.180265 \\
\hline 10 & 1 & 0 & 0.013368 & -0.321419 & 2.106726 \\
\hline 11 & 1 & 0 & -0.378579 & 0.984537 & 0.971277 \\
\hline 12 & 1 & 0 & -2.380875 & -0.338822 & 1.817334 \\
\hline 13 & 1 & 0 & -1.690677 & -1.775294 & 1.096185 \\
\hline 14 & 1 & 0 & -3.387191 & 1.304505 & -1.378398 \\
\hline 15 & 1 & 0 & -2.062280 & 1.773572 & -0.312385 \\
\hline 16 & 1 & 0 & -3.634138 & 1.337973 & 0.359683 \\
\hline
\end{tabular}




$\begin{array}{rrrrrr}17 & 8 & 0 & -0.067733 & 2.841928 & 0.190297 \\ 18 & 1 & 0 & -0.072667 & 3.750434 & 0.463292 \\ 19 & 1 & 0 & 1.450427 & 1.907144 & -0.629632 \\ 20 & 1 & 0 & 3.113282 & 1.431180 & -1.125424 \\ 21 & 1 & 0 & 1.707152 & 0.878236 & -2.053316 \\ 22 & 1 & 0 & -1.810144 & -0.581768 & -1.148402 \\ 23 & 6 & 0 & -3.717053 & -1.288767 & -0.506766 \\ 24 & 1 & 0 & -4.431621 & -1.145012 & 0.302762 \\ 25 & 1 & 0 & -4.235153 & -1.080667 & -1.440291 \\ 26 & 1 & 0 & -3.431219 & -2.338512 & -0.512391\end{array}$

Total energy : $\quad-900.782064533$

Number of imaginary frequencies: 0

\section{TS1-4A}

\begin{tabular}{|c|c|c|c|c|c|}
\hline \multirow{2}{*}{$\begin{array}{l}\text { Center } \\
\text { Number }\end{array}$} & \multirow{2}{*}{$\begin{array}{l}\text { Atomic } \\
\text { Number }\end{array}$} & \multirow{2}{*}{$\begin{array}{l}\text { Atomic } \\
\text { Type }\end{array}$} & \multicolumn{3}{|c|}{ Coordinates (Angstroms) } \\
\hline & & & $x$ & $\mathrm{Y}$ & Z \\
\hline 1 & 15 & 0 & -1.836191 & -0.174918 & 0.031009 \\
\hline 2 & 9 & 0 & -2.436706 & -1.566573 & 0.588111 \\
\hline 3 & 6 & 0 & -2.047901 & 0.732044 & 1.567930 \\
\hline 4 & 8 & 0 & -0.359842 & -0.705460 & -0.109304 \\
\hline 5 & 6 & 0 & 0.506520 & -0.412781 & -1.207162 \\
\hline 6 & 6 & 0 & 1.909169 & -0.859825 & -0.829703 \\
\hline 7 & 6 & 0 & 2.536451 & -0.129532 & 0.366708 \\
\hline 8 & 6 & 0 & 2.698631 & 1.372560 & 0.113083 \\
\hline 9 & 8 & 0 & -2.600472 & 0.149957 & -1.168006 \\
\hline 10 & 1 & 0 & 0.159052 & -0.978226 & -2.065650 \\
\hline 11 & 1 & 0 & 0.436959 & 0.645061 & -1.400127 \\
\hline 12 & 1 & 0 & 2.542618 & -0.716945 & -1.707777 \\
\hline 13 & 1 & 0 & 1.893263 & -1.930296 & -0.628166 \\
\hline 14 & 1 & 0 & 3.178228 & 1.849162 & 0.967299 \\
\hline 15 & 1 & 0 & 1.740983 & 1.855908 & -0.051966 \\
\hline 16 & 1 & 0 & 3.336584 & 1.547071 & -0.755173 \\
\hline 17 & 8 & 0 & -0.526992 & 2.141702 & -0.329474 \\
\hline 18 & 1 & 0 & -1.062376 & 2.596165 & -0.967715 \\
\hline 19 & 1 & 0 & -1.621946 & 1.714193 & 1.439599 \\
\hline 20 & 1 & 0 & -3.111207 & 0.776869 & 1.784553 \\
\hline 21 & 1 & 0 & -1.553344 & 0.188303 & 2.366303 \\
\hline 22 & 1 & 0 & 1.865953 & -0.253760 & 1.212701 \\
\hline 23 & 6 & 0 & 3.878554 & -0.776005 & 0.729053 \\
\hline 24 & 1 & 0 & 4.590680 & -0.685353 & -0.090377 \\
\hline 25 & 1 & 0 & 4.321136 & -0.298549 & 1.600515 \\
\hline
\end{tabular}


Total energy : $\quad-900.946223290$

Number of imaginary frequencies: 1

\section{Reactant-5}

\begin{tabular}{|c|c|c|c|c|c|}
\hline \multirow{2}{*}{$\begin{array}{l}\text { Center } \\
\text { Number }\end{array}$} & \multirow{2}{*}{$\begin{array}{l}\text { Atomic } \\
\text { Number }\end{array}$} & \multirow{2}{*}{$\begin{array}{l}\text { Atomic } \\
\text { Type }\end{array}$} & \multicolumn{3}{|c|}{ Coordinates (Angstroms) } \\
\hline & & & $x$ & $\mathrm{Y}$ & Z \\
\hline 1 & 15 & 0 & -1.845328 & -0.450321 & 0.108455 \\
\hline 2 & 6 & 0 & -2.956529 & -1.003740 & -1.169374 \\
\hline 3 & 8 & 0 & -1.205781 & -1.435513 & 0.966011 \\
\hline 4 & 8 & 0 & -0.792775 & 0.481177 & -0.631894 \\
\hline 5 & 6 & 0 & 0.146925 & 1.270337 & 0.101490 \\
\hline 6 & 6 & 0 & 1.565268 & 0.979912 & -0.382573 \\
\hline 7 & 7 & 0 & 2.213676 & -0.343154 & -0.030564 \\
\hline 8 & 6 & 0 & 3.665513 & -0.213008 & -0.375081 \\
\hline 9 & 6 & 0 & 1.650157 & -1.486242 & -0.822508 \\
\hline 10 & 6 & 0 & 2.094119 & -0.655686 & 1.431494 \\
\hline 11 & 9 & 0 & -2.682041 & 0.583679 & 0.917292 \\
\hline 12 & 1 & 0 & -3.367172 & -0.160657 & -1.709767 \\
\hline 13 & 1 & 0 & -3.761944 & -1.564790 & -0.709934 \\
\hline 14 & 1 & 0 & -2.429884 & -1.653596 & -1.857343 \\
\hline 15 & 1 & 0 & 0.054583 & 1.053485 & 1.156550 \\
\hline 16 & 1 & 0 & 2.210898 & 1.736541 & 0.039804 \\
\hline 17 & 1 & 0 & 1.603208 & 1.064168 & -1.459478 \\
\hline 18 & 1 & 0 & 4.158038 & -1.152548 & -0.178666 \\
\hline 19 & 1 & 0 & 4.108445 & 0.562900 & 0.230599 \\
\hline 20 & 1 & 0 & 3.761001 & 0.036819 & -1.421275 \\
\hline 21 & 1 & 0 & 2.271058 & -2.352131 & -0.648640 \\
\hline 22 & 1 & 0 & 1.667351 & -1.228043 & -1.870550 \\
\hline 23 & 1 & 0 & 0.650291 & -1.693275 & -0.490472 \\
\hline 24 & 1 & 0 & 2.721777 & -1.507295 & 1.646656 \\
\hline 25 & 1 & 0 & 1.070845 & -0.897997 & 1.667042 \\
\hline 26 & 1 & 0 & 2.434181 & 0.196843 & 2.001563 \\
\hline 27 & 6 & 0 & -0.174691 & 2.739720 & -0.140992 \\
\hline 28 & 1 & 0 & 0.486958 & 3.380144 & 0.431628 \\
\hline 29 & 1 & 0 & -0.081999 & 2.987644 & -1.192345 \\
\hline 30 & 1 & 0 & -1.191196 & 2.942498 & 0.168454 \\
\hline
\end{tabular}

Total energy: $\quad-919.784062200$

Number of imaginary frequencies: 0 


\section{HBR-5A}

\begin{tabular}{|c|c|c|c|c|c|}
\hline \multirow{2}{*}{$\begin{array}{l}\text { Center } \\
\text { Number }\end{array}$} & \multirow{2}{*}{$\begin{array}{l}\text { Atomic } \\
\text { Number }\end{array}$} & \multirow{2}{*}{$\begin{array}{l}\text { Atomic } \\
\text { Type }\end{array}$} & \multicolumn{3}{|c|}{ Coordinates (Angstroms) } \\
\hline & & & $x$ & $\mathrm{Y}$ & Z \\
\hline 1 & 15 & 0 & -2.182301 & -0.087950 & -0.184044 \\
\hline 2 & 9 & 0 & -2.819676 & 0.226564 & -1.583316 \\
\hline 3 & 6 & 0 & -2.075786 & -1.868434 & -0.192782 \\
\hline 4 & 8 & 0 & -0.738263 & 0.498629 & -0.483060 \\
\hline 5 & 6 & 0 & 0.175577 & 0.981786 & 0.517471 \\
\hline 6 & 6 & 0 & 1.512629 & 1.146381 & -0.202648 \\
\hline 7 & 7 & 0 & 2.405155 & -0.063565 & -0.343115 \\
\hline 8 & 6 & 0 & 3.515431 & 0.317347 & -1.252702 \\
\hline 9 & 6 & 0 & 1.679690 & -1.245019 & -0.920521 \\
\hline 10 & 6 & 0 & 2.973698 & -0.456926 & 0.992171 \\
\hline 11 & 8 & 0 & -2.905599 & 0.533497 & 0.909584 \\
\hline 12 & 1 & 0 & 0.230861 & 0.243918 & 1.308053 \\
\hline 13 & 1 & 0 & 2.112498 & 1.879110 & 0.318486 \\
\hline 14 & 1 & 0 & 1.321060 & 1.510561 & -1.202597 \\
\hline 15 & 1 & 0 & 4.207352 & -0.507718 & -1.329559 \\
\hline 16 & 1 & 0 & 4.027703 & 1.181513 & -0.854227 \\
\hline 17 & 1 & 0 & 3.117249 & 0.548075 & -2.230208 \\
\hline 18 & 1 & 0 & 2.422399 & -1.993373 & -1.161441 \\
\hline 19 & 1 & 0 & 1.167885 & -0.928571 & -1.817028 \\
\hline 20 & 1 & 0 & 1.004978 & -1.629118 & -0.167031 \\
\hline 21 & 1 & 0 & 3.706339 & -1.233295 & 0.820795 \\
\hline 22 & 1 & 0 & 2.162549 & -0.850179 & 1.603489 \\
\hline 23 & 1 & 0 & 3.457322 & 0.409734 & 1.422600 \\
\hline 24 & 8 & 0 & 0.441831 & -1.706553 & 1.749120 \\
\hline 25 & 1 & 0 & 0.209425 & -2.201942 & 2.523064 \\
\hline 26 & 1 & 0 & -1.364030 & -2.158179 & 0.576430 \\
\hline 27 & 1 & 0 & -3.056968 & -2.278872 & 0.017194 \\
\hline 28 & 1 & 0 & -1.738793 & -2.222915 & -1.160422 \\
\hline 29 & 6 & 0 & -0.272877 & 2.343793 & 1.032573 \\
\hline 30 & 1 & 0 & -0.299591 & 3.079903 & 0.232957 \\
\hline 31 & 1 & 0 & -1.259644 & 2.269123 & 1.464460 \\
\hline 32 & 1 & 0 & 0.413004 & 2.689208 & 1.800777 \\
\hline
\end{tabular}

Total energy : -995.337805448

Number of imaginary frequencies: 0

\section{TS1-5A}

Center Atomic Atomic Coordinates (Angstroms)




\begin{tabular}{|c|c|c|c|c|c|}
\hline Number & Number & Type & $X$ & $Y$ & Z \\
\hline 1 & 15 & 0 & -1.986123 & -0.162624 & -0.013012 \\
\hline 2 & 9 & 0 & -2.861966 & 0.517448 & -1.174953 \\
\hline 3 & 6 & 0 & -2.264801 & -1.845046 & -0.588810 \\
\hline 4 & 8 & 0 & -0.607210 & 0.444496 & -0.562934 \\
\hline 5 & 6 & 0 & 0.257040 & 1.178274 & 0.279365 \\
\hline 6 & 6 & 0 & 1.662737 & 1.074629 & -0.303618 \\
\hline 7 & 7 & 0 & 2.412983 & -0.228814 & -0.156775 \\
\hline 8 & 6 & 0 & 3.754298 & -0.025166 & -0.762109 \\
\hline 9 & 6 & 0 & 1.729450 & -1.363940 & -0.866305 \\
\hline 10 & 6 & 0 & 2.572115 & -0.596036 & 1.289857 \\
\hline 11 & 8 & 0 & -2.501515 & 0.343289 & 1.251004 \\
\hline 12 & 1 & 0 & 0.208362 & 0.758994 & 1.269373 \\
\hline 13 & 1 & 0 & 2.290348 & 1.817742 & 0.169617 \\
\hline 14 & 1 & 0 & 1.622533 & 1.285002 & -1.364054 \\
\hline 15 & 1 & 0 & 4.325804 & -0.936289 & -0.669278 \\
\hline 16 & 1 & 0 & 4.265901 & 0.775020 & -0.247031 \\
\hline 17 & 1 & 0 & 3.642810 & 0.226372 & -1.807018 \\
\hline 18 & 1 & 0 & 2.420249 & -2.196039 & -0.883373 \\
\hline 19 & 1 & 0 & 1.503407 & -1.050711 & -1.875094 \\
\hline 20 & 1 & 0 & 0.841453 & -1.624464 & -0.311550 \\
\hline 21 & 1 & 0 & 3.255556 & -1.432004 & 1.345441 \\
\hline 22 & 1 & 0 & 1.594401 & -0.884471 & 1.665123 \\
\hline 23 & 1 & 0 & 2.992795 & 0.251947 & 1.813776 \\
\hline 24 & 8 & 0 & -0.280345 & -1.406342 & 1.296771 \\
\hline 25 & 1 & 0 & -0.750179 & -1.601322 & 2.096521 \\
\hline 26 & 1 & 0 & -1.996550 & -2.557304 & 0.169811 \\
\hline 27 & 1 & 0 & -3.311656 & -1.926811 & -0.858740 \\
\hline 28 & 1 & 0 & -1.669464 & -2.022748 & -1.477890 \\
\hline 29 & 6 & 0 & -0.155586 & 2.647248 & 0.292559 \\
\hline 30 & 1 & 0 & -0.113912 & 3.079143 & -0.703247 \\
\hline 31 & 1 & 0 & -1.170178 & 2.727332 & 0.658329 \\
\hline 32 & 1 & 0 & 0.488019 & 3.223313 & 0.952108 \\
\hline
\end{tabular}

HF/6-31+G(d) energy : -995.334053680

Number of imaginary frequencies: 1

\section{Reactant-6}

\begin{tabular}{cccccc} 
Center & Atomic & Atomic & \multicolumn{4}{c}{ Coordinates } & (Angstroms) \\
Number & Number & Type & X & $Y$ & Z \\
-15 & 15 & 0 & -1.671921 & -0.299896 & 0.227237
\end{tabular}




\begin{tabular}{rrrrrr}
2 & 6 & 0 & -2.134872 & -1.808742 & -0.615069 \\
3 & 8 & 0 & -1.604388 & -0.327220 & 1.676810 \\
4 & 8 & 0 & -0.380566 & 0.186445 & -0.517206 \\
5 & 6 & 0 & 0.575126 & 1.123402 & 0.035634 \\
6 & 6 & 0 & 1.931837 & 0.825541 & -0.605072 \\
7 & 7 & 0 & 2.630658 & -0.380963 & -0.228052 \\
8 & 6 & 0 & 2.113430 & -1.629938 & -0.739570 \\
9 & 6 & 0 & 3.025628 & -0.457901 & 1.159987 \\
10 & 9 & 0 & -2.765872 & 0.699199 & -0.289376 \\
11 & 1 & 0 & -2.155770 & -1.657434 & -1.686972 \\
12 & 1 & 0 & -3.117510 & -2.114477 & -0.275681 \\
13 & 1 & 0 & -1.424243 & -2.589795 & -0.375774 \\
14 & 1 & 0 & 0.608761 & 0.957965 & 1.102999 \\
15 & 1 & 0 & 2.581844 & 1.662529 & -0.365795 \\
16 & 1 & 0 & 1.802678 & 0.825556 & -1.682531 \\
17 & 1 & 0 & 2.891718 & -2.384527 & -0.684886 \\
18 & 1 & 0 & 1.830051 & -1.514974 & -1.778795 \\
19 & 1 & 0 & 1.248888 & -2.004371 & -0.190132 \\
20 & 1 & 0 & 3.760888 & -1.246062 & 1.280962 \\
21 & 1 & 0 & 2.198531 & -0.668914 & 1.841607 \\
22 & 1 & 0 & 3.487860 & 0.474884 & 1.465006 \\
23 & 6 & 0 & 0.110515 & 2.540870 & -0.259573 \\
24 & 1 & 0 & 0.808124 & 3.255759 & 0.164742 \\
25 & 1 & 0 & 0.049723 & 2.711831 & -1.329572 \\
26 & 1 & 0 & -0.864841 & 2.731223 & 0.172158 \\
& 1 & & & - & \\
\hline & & 0 & &
\end{tabular}

Total energy : -880.371109348

Number of imaginary frequencies: 0

\section{HBR-6A}

\begin{tabular}{|c|c|c|c|c|c|}
\hline \multirow{2}{*}{$\begin{array}{l}\text { Center } \\
\text { Number }\end{array}$} & \multirow{2}{*}{$\begin{array}{l}\text { Atomic } \\
\text { Number }\end{array}$} & \multirow{2}{*}{$\begin{array}{l}\text { Atomic } \\
\text { Type }\end{array}$} & \multicolumn{3}{|c|}{ Coordinates (Angstroms) } \\
\hline & & & $x$ & $\mathrm{Y}$ & Z \\
\hline 1 & 15 & 0 & -1.868142 & -0.125228 & -0.154232 \\
\hline 2 & 9 & 0 & -2.440776 & -0.365716 & -1.608526 \\
\hline 3 & 6 & 0 & -1.582857 & -1.779970 & 0.446242 \\
\hline 4 & 8 & 0 & -0.500994 & 0.515911 & -0.579886 \\
\hline 5 & 6 & 0 & 0.529333 & 1.030238 & 0.317519 \\
\hline 6 & 6 & 0 & 1.798420 & 1.169719 & -0.530206 \\
\hline 7 & 7 & 0 & 2.600705 & -0.002143 & -0.817385 \\
\hline 8 & 6 & 0 & 1.949045 & -1.019266 & -1.613842 \\
\hline 9 & 6 & 0 & 3.250573 & -0.566124 & 0.358796 \\
\hline 10 & 8 & 0 & -2.782015 & 0.703670 & 0.622890 \\
\hline
\end{tabular}




\begin{tabular}{rrrrrr}
11 & 1 & 0 & 0.657079 & 0.321426 & 1.129015 \\
12 & 1 & 0 & 2.449402 & 1.871720 & -0.015859 \\
13 & 1 & 0 & 1.518023 & 1.632745 & -1.473612 \\
14 & 1 & 0 & 2.703261 & -1.704046 & -1.995185 \\
15 & 1 & 0 & 1.448936 & -0.562208 & -2.460786 \\
16 & 1 & 0 & 1.217097 & -1.603236 & -1.059227 \\
17 & 1 & 0 & 3.944559 & -1.337757 & 0.032743 \\
18 & 1 & 0 & 2.556472 & -0.988110 & 1.083047 \\
19 & 1 & 0 & 3.834949 & 0.210723 & 0.846494 \\
20 & 8 & 0 & 0.679337 & -1.268458 & 2.388161 \\
21 & 1 & 0 & 0.707740 & -1.422142 & 3.323967 \\
22 & 1 & 0 & -0.862674 & -1.739952 & 1.275930 \\
23 & 1 & 0 & -2.527843 & -2.195191 & 0.781904 \\
24 & 1 & 0 & -1.179484 & -2.397681 & -0.348757 \\
25 & 6 & 0 & 0.087584 & 2.385117 & 0.851063 \\
26 & 1 & 0 & -0.013770 & 3.110422 & 0.045822 \\
27 & 1 & 0 & -0.861583 & 2.308841 & 1.363736 \\
28 & 1 & 0 & 0.828807 & 2.752037 & 1.555919 \\
\hline------------------------------------------------------ & &
\end{tabular}

Total energy : -955.791968899

Number of imaginary frequencies: 0

\section{TS1-6A}

\begin{tabular}{cccccc} 
Center & Atomic & Atomic & \multicolumn{3}{c}{ Coordinates (Angstroms) } \\
Number & Number & Type & $X$ & $Y$ & $Z$ \\
-15 & 15 & 0 & -1.729782 & -0.128747 & -0.004685 \\
1 & 9 & 0 & -2.610200 & 0.298980 & -1.285327 \\
2 & 6 & 0 & -1.861824 & -1.899812 & -0.274037 \\
3 & 8 & 0 & -0.371788 & 0.431943 & -0.569395 \\
4 & 6 & 0 & 0.634000 & 1.133634 & 0.183407 \\
5 & 6 & 0 & 1.946340 & 1.014474 & -0.598005 \\
6 & 7 & 0 & 2.698450 & -0.223863 & -0.543553 \\
7 & 6 & 0 & 2.044306 & -1.369138 & -1.150950 \\
8 & 6 & 0 & 3.187882 & -0.548579 & 0.787262 \\
9 & 8 & 0 & -2.347308 & 0.503559 & 1.155571 \\
10 & 1 & 0 & 0.698479 & 0.640877 & 1.140493 \\
11 & 1 & 0 & 2.608792 & 1.797078 & -0.233704 \\
12 & 1 & 0 & 1.739093 & 1.239968 & -1.641584 \\
13 & 1 & 0 & 2.786058 & -2.151031 & -1.304683 \\
14 & 1 & 0 & 1.647513 & -1.090592 & -2.122064 \\
15 & 1 & 0 & 1.241655 & -1.769326 & -0.540082 \\
16 & 1 & 0 & 3.918525 & -1.350639 & 0.704314 \\
17 & & & & &
\end{tabular}




$\begin{array}{rrrrrr}18 & 1 & 0 & 2.394910 & -0.863866 & 1.461249 \\ 19 & 1 & 0 & 3.700924 & 0.316014 & 1.203321 \\ 20 & 8 & 0 & 0.039194 & -1.251697 & 1.729420 \\ 21 & 1 & 0 & -0.404548 & -1.141747 & 2.561154 \\ 22 & 1 & 0 & -1.258614 & -2.398086 & 0.468185 \\ 23 & 1 & 0 & -2.910305 & -2.169784 & -0.188993 \\ 24 & 1 & 0 & -1.522920 & -2.132458 & -1.278117 \\ 25 & 6 & 0 & 0.216307 & 2.592276 & 0.320774 \\ 26 & 1 & 0 & 0.146321 & 3.074537 & -0.652447 \\ 27 & 1 & 0 & -0.745596 & 2.664851 & 0.812065 \\ 28 & 1 & 0 & 0.946236 & 3.134065 & 0.918500\end{array}$

Total energy : -955.787848240

Number of imaginary frequencies: 1 